clinically uninvolved skin from patients with SSc and b) very early disease at the puffy/edematous phase, prior to skin thickening.

Methods: Skin biopsies were obtained from a) 12 patients with SSc from lesional skin of the forearm, b) 5 patients with SSc from the upper back which was not affected clinically, c) 2 patients with systemic sclerosis (SSc) with very early disease (<12 months). These patients had puffy hands but no skin thickening; the biopsy was obtained from the distal part of the forearm, in close proximity to the edematous area d) 5 healthy subjects. Dkk- 1 expression was immunohistochemically assessed using a mouse anti-human monoclonal antibody ( $R$ and $D$ Systems) by a semi-quantitative method (high/moderate/weak/no expression).

Results: Healthy skin displayed a high Dkk-1 immunoexpression in basal cells of the epidermis as well as in the fibroblasts of the dermis in sharp contrast to clinically involved scleroderma skin that displayed no Dkk-1 immunoexpression. Clinically uninvolved skin was obtained from 5 patients with SSc (4 diffuse- 1 limited) with a median age of $50^{46-72}$ and disease duration of $6^{2-13}$ years. In all 5 biopsies Dkk-1 was only moderately expressed in basal cells of the epidermis and dermal fibroblasts. Clinically uninvolved scleroderma skin could by differentiated by immunohistochemical means from both skin from healthy subjects (high Dkk-1 expression) and clinically involved scleroderma skin (no Dkk-1 expression). Skin from very early disease at the edematous phase, prior to skin thickening, displayed only a weak Dkk-1 immunoreactivity in basal cells of the epidermis as well as in the fibroblasts of the dermis.

Conclusions: The decrease in Dkk-1 expression in clinically uninvolved scleroderma skin substantiates previous evidence that the skin in SSc is universally affected underling the systemic nature of the disease. The downregulation of Dkk1 at the edematous phase of the disease indicates that the Wnt pathway is involved early in the disease process, prior to establishment of fibrosis a finding with potential pathogenetic implications

REFERENCES:

[1] Daoussis, et al. ART 2008.

[2] Akhmetshina, et al. Nat Commun 2012.

Disclosure of Interest: None declared

DOI: 10.1136/annrheumdis-2018-eular.5464
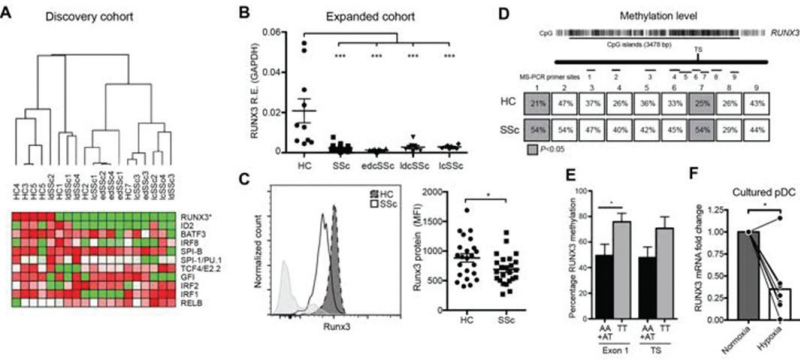

G

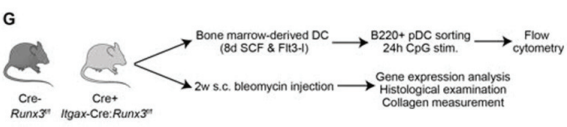

H
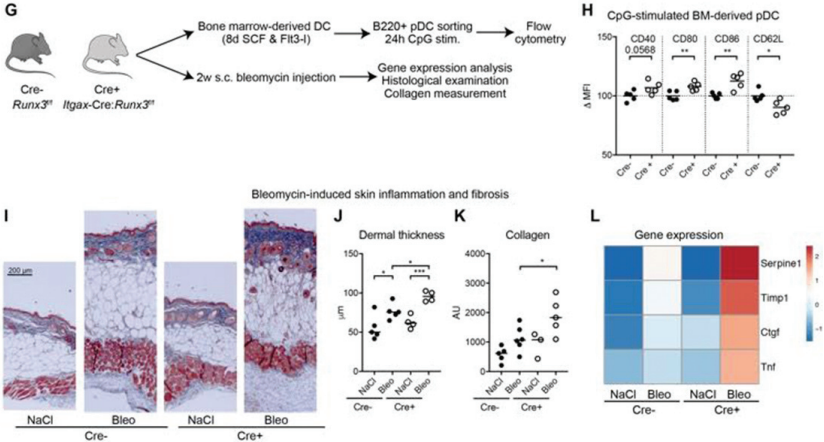

Conclusions: We found low RUNX3 expression in pDCs of SSc patients. The presence of a SNP and higher methylation status of RUNX3, and downregulation in hypoxic condition, suggest at least three pathways underlying the low RUNX3 expression observed in SSc pDCs. We demonstrate a detrimental role of RUNX3 ablated DCs in a mouse SSc model further underscoring the role of pDCs in this disease. Further research is warranted to explore the potential therapeutic effect of RUNX3 targeting in fibrotic disease.

Disclosure of Interest: None declared

DOI: 10.1136/annrheumdis-2018-eular.6538

\section{OP0094 SLIT2/ROBO4 AXIS MAY CONTRIBUTE TO ANGIOGENESIS DISTURBANCE IN SYSTEMIC SCLEROSIS (SSC)}

W. Marut ${ }^{1}$, A. Affandi ${ }^{1}$, J. Broen ${ }^{1}$, L. Bossini-Castillo ${ }^{2}$, A. Ottria ${ }^{1}$, R. Tieland ${ }^{1}$, L. van Bon $^{1}$, M. Rossato ${ }^{1}$, L. de Kroon ${ }^{3}$, J. van Roon ${ }^{1}$, J. Martin², R. Lafyatis ${ }^{3}$, T. Radstake ${ }^{1} .1$ Laboratory for translational immunology, University Medical Center Utrecht, Utrecht, Netherlands; ${ }^{2}$ Consejo Superior de Investigaciones Científicas, Instituto de Parasitología y Biomedicina López-Neyra, Granada, Spain;

${ }^{3}$ Rheumatology Section, Department of Medicine, Boston University School of Medicine, Boston, USA

Background: Systemic sclerosis (SSc) is an autoimmune disease with unknown pathogenesis manifested by inflammation, vasculopathy, and fibrosis in skin and internal organs. The type I IFN signature found in SSc propelled us to study plasmacytoid dendritic cells ( $p D C s$ ) in this disease.

Objectives: To identify candidate pathways underlying pDC aberrancies in SSc and to validate its function on pDC biology.

Methods: PCR-based transcription factor profiling and methylation status analyses, SNP genotyping by sequencing, and flow cytometry analysis were performed in pDCs from healthy controls or SSc patients. pDCs were also cultured under hypoxia and RUNX3 levels were determined. To study Runx3 function in DCs, Itgax-Cre:Run $\times 3^{\text {t/f }}$ mice were used in an in vitro functional assays and bleomycininduced SSc skin inflammation and fibrosis model.

Results: Transcription factor RUNX3 was significantly downregulated in SSc pDCs on RNA and protein levels. A higher methylation status of the RUNX3 gene correlated with RUNX3 gene expression level and disease susceptibility. After sequencing of the RUNX3 promoter region, we identified a non-synonymous SNP rs6672420 associated with SSc and hypermethylation of RUNX3. Additionally, pDCs cultured in hypoxic conditions showed a significantly lower RUNX3 expression. Furthermore, mouse pDCs deficient of Runx3 showed enhanced expression of co-stimulatory molecules upon CpG stimulation. Finally, in SSc bleomycin model, mice with DC-specific deletion of Runx3 showed increased skin inflammation and fibrosis. 
$6.68-12.13 \mathrm{ng} / \mathrm{ml})(\mathrm{p}=0.002$ and $\mathrm{p}=0.001$, respectively). Serum Slit2 was elevated in SSc patients irrespective of the nailfold videocapillaroscopy (NVC) pattern or the presence/absence of digital ulcers. Interestingly, differences in serum Slit2 levels were found between VEDOSS patients with early/active NVC pattern and controls $(p<0.0005)$, while Slit2 concentrations were similar in VEDOSS with normal NVC and controls. In SSc, Slit2 and Robo4 expression was higher in clinically affected skin and cultured MVECs in respect to controls. No difference was found in Robo1 expression. Cell viability, wound healing capacity and capillarylike tube formation in H-MVECs were all significantly reduced after challenge with rh Slit2 or SSc sera. These inhibitory effects were significantly attenuated when SSc sera were preincubated with anti-Slit2 blocking antibody. Cell viability, wound healing capacity and in vitro angiogenesis were severely compromised in SScMVECs and could be significantly ameliorated by Slit2 neutralisation or Robo4 gene silencing.

Conclusions: In SSc, increased circulating levels of Slit2 and activation of the Slit2/Robo4 antiangiogenic axis may contribute to peripheral microangiopathy since the very early phase of the disease.

Disclosure of Interest: None declared

DOI: 10.1136/annrheumdis-2018-eular.4610

\section{OP0095 SOLUBLE CD163 AS A POTENTIAL BIOMARKER IN SYSTEMIC SCLEROSIS}

C. Frantz, S. Pezet, J. Avouac, Y. Allanore. INSERM U1016, UMR8104, Paris

Descartes University, Rheumatology A department, Cochin Hospital, Paris, France

Background: Recent accumulating evidences indicate a crucial role of macrophage lineage in the pathogenesis of fibrotic diseases including systemic sclerosis (SSc). CD163 is a surface marker expressed by M2 macrophages that accumulate during the healing phase of acute inflammation. It is actively released from the plasma membrane in response to certain inflammatory stimuli and enters the circulation in its soluble form (sCD163).

Objectives: In this study, we aimed to evaluate the performance of serum and urinary sCD163 concentrations as possible biomarker in SSc.

Methods: Urine and serum samples were obtained from SSc patients, fulfilling the 2013 ACR/EULAR classification criteria for SSc, and age- and sex-matched controls. Serum and urinary SCD163 concentrations were measured by commercially available ELISA kit ( $R$ and $D$ systems) and evaluated for their significance as potential biomarkers. Statistical analysis was carried out using Mann-Whitney $U$ test and the relationship between parameters was statistically examined by Spearman's rank test.

Results: Two hundred and three SSc patients were included, $163(80 \%)$ were female, with a mean \pm standard deviation (SD) age of $59 \pm 13$ years and a mean $\pm S D$ disease duration of $12 \pm 9$ years. Eighty-one (41\%) patients had diffuse cutaneous SSc and mean \pm SD mRSS was 6.6 \pm 7.7 . Lung fibrosis on imaging was observed in $33 \%$ of the patients, $7 \%$ had pulmonary arterial hypertension, $44 \%$ had history of digital ulcers and $41 \%$ were taking immunosuppressive therapy. Control group consisted of 47 age- and sex-matched patients with non-inflammatory diseases, being osteoporosis for the very large majority.

Serum SCD163 levels were significantly higher in SSc patients compared with controls (mean $\pm S D$ : $529 \pm 251$ vs $385.1 \pm 153 \mathrm{ng} / \mathrm{ml}$; $<0.001$ ). Urinary sCD163 concentrations in SSc patients were also higher than those in controls, but this did not reach significance $(236.9 \pm 498$ vs $176.2 \pm 173 \mathrm{ng} / \mathrm{mg} \mathrm{uCr} ; \mathrm{p}=0.580)$. When looking at the subsets according to skin disease or disease duration, no difference could be identified. Furthermore, when the organ involvements were investigated, no subpopulation could be identified as having higher concentrations.

Conclusions: To our knowledge this is the first evaluation of both serum and urinary SCD163 levels in SSc. Our results show a significant difference for sera values that should be prioritised for further studies as compared to urinary concentrations conversely to what has been described in lupus. Our results further support that the M2 macrophages/CD163 signalling system may play a role in the pathogenesis of SSc. However, further studies are required to address the exact role of CD163 in the pathogenesis of SSc and to determine whether it could help in the risk-stratification of the patients in this heterogeneous disease.

Disclosure of Interest: None declared

DOI: 10.1136/annrheumdis-2018-eular.2631
WEDNESDAY, 13 JUNE 2018

\section{A rollercoaster from bench to bedside and back again: paediatric rheumatology in the 21 st century}

\begin{tabular}{l|l}
\hline OP0096 & ADENO-ASSOCIATED VIRUS VECTOR-MEDIATED \\
INTERLEUKIN-10 INDUCTION PREVENTS VASCULAR \\
INFLAMMATION IN A MURINE MODEL OF KAWASAKI \\
DISEASE
\end{tabular}

J Nakamura $^{1,2}$, S. Watanabe ${ }^{1}$, H. Kimura ${ }^{1}$, H. Mizukami ${ }^{3}$, N. Nagi-Miura ${ }^{4}$, N. Ohno ${ }^{4}$, M. Takahashi ${ }^{1}$, S. Minota ${ }^{2} .{ }^{1}$ Division of Inflammation Research; ${ }^{2}$ Rheumatology and Clinical Immunology, ${ }^{3}$ Division of Genetic Therapeutics, Jichi Medical University, Shimotsuke, Tochigi; ${ }^{4}$ Laboratory for Immunopharmacology of Microbial Products, School of Pharmacy, Tokyo University of Pharmacy and Life Sciences, Hachioji, Tokyo, Japan

Background: Kawasaki disease (KD), which is a common paediatric heart disease, is characterised by coronary vasculitis and subsequently aneurysm formation. Although the administration of intravenous immunoglobulin (IVIG) is effective for reducing aneurysm formation, approximately $10 \%-20 \%$ of patients are resistant to this therapy. Therefore, additional therapeutic approaches for treating the IVIG-resistant patients need to be developed.

Candida albicans water-soluble fraction (CAWS)-induced vasculitis on coronary arteries and root of aorta is a frequently used murine model of KD. It has been considered that C-type lectin receptor Dectin-2 recognises CAWS. Recent studies showed CAWS-resistant strains of mice have higher serum IL-10 levels, which suggested that IL-10 might negatively regulate the development of CAWS induced vasculitis.

Objectives: The aim of the study is to investigate the therapeutic effect of IL-10 in CAWS-induced vasculitis and elucidate the underlying pathogenesis of KD.

Methods: To induce the expression of IL-10 in vivo, Adeno-associated virus (AAV) vectors encoding IL-10 were injected into DBA/2 mice. After the induction of IL-10, the mice were treated intraperitoneally with CAWS to induce vasculitis. Cardiac functions by echocardiography, inflammation and fibrosis by histological analyses, gene expression of inflammatory cytokines and fibrosis-related factors in the heart, and infiltrating cells by flow cytometry were assessed to evaluate the effects of IL- 10 .

For in vitro study, bone marrow-derived macrophages (BMDM) were stimulated with CAWS in presence or absence of IL-10. TNF- $\alpha$ and IL- 6 produced by the BMDM and Dectin-2 expressions on the BMDM were assessed.

Results: AAV-mediated induction of IL-10 significantly attenuated CAWSinduced cardiac functions (\%FS and LVEDD). Histological analyses revealed that $\mathrm{IL}-10$ markedly attenuated the vascular inflammation and fibrosis in the aortic root and coronary artery. Accordingly, increased gene expressions of inflammatory cytokines or fibrosis-related factors in the heart of CAWS-treated mice were significantly reduced by $\mathrm{IL}-10$. The predominant infiltrating inflammatory cells in vascular walls were Dectin- $2^{+} \mathrm{CD} 11 \mathrm{~b}^{+}$macrophages, and they were also decreased by IL-10.

Furthermore, we showed GM-CSF induced Dectin-2 expression on BMDM, and the GM-CSF-treated BMDM produced TNF- $\alpha$ and IL- 6 upon CAWS-stimulation. $\mathrm{IL}-10$ had no effect on the Dectin-2 expression but significantly inhibited the production of the cytokines. Finally, the AAV-mediated induction of IL-10 prevented the expression of TNF- $\alpha$ and IL- 6 in the heart of the mice treated with CAWS for 24 hours (at the early phase), but not GM-CSF and Dectin-2. These results suggest that GM-CSF mediates CAWS-induced vasculitis via Dectin-2 upregulation and IL-10 inhibits the downstream of GM-CSF and Dectin-2 signalling.

Conclusions: Our study has shown that IL-10 may have therapeutic application in the prevention of coronary vasculitis and aneurysm formation, and provided new insights into the mechanism underlying the pathogenesis of KD.

Disclosure of Interest: None declared

DOI: 10.1136/annrheumdis-2018-eular.2086

\section{OP0097 STIMULATED MONOCYTE-DERIVED MACROPHAGES FROM PATIENTS WITH ENTHESITIS RELATED ARTHRITIS SECRETE HIGHER LEVELS OF IL23 AND LOWER LEVELS OF INTERFERON GAMMA COMPARED TO HEALTHY CONTROLS}

C Fisher ${ }^{1,2}$, D. Eleftheriou ${ }^{1}$, D. Sen ${ }^{1,2}$, Y. Ioannou ${ }^{1} .{ }^{1}$ Arthritis Research UK Centre for Adolescent Rheumatology, University College London; ${ }^{2}$ National Institute for Health Research University College London Hospitals Biomedical Research Centre, University College London and University College London Hospital, London, UK

Background: Enthesitis related arthritis (ERA) is the subtype of juvenile idiopathic arthritis most closely related to adult spondyloarthropathy (SpA) 